\title{
Impacto da retirada do corpo adiposo da bochecha em estruturas anatômicas
}

\section{associadas: uma revisão integrativa}

Impact of the removal of the buccal fat pad on associated anatomical structures: a integrative

review

Impacto de la extracción del cuerpo adiposo de la mejilla en estructuras anatómicas asociadas: una revisión integradora

\section{Resumo}

A presente revisão de literatura teve o objetivo de estudar as complicações que decorrem da retirada cirúrgica do corpo adiposo da bochecha, buscando entender as relações anatômicas entre as estruturas envolvidas nesse procedimento e observar as principais sequelas acarretadas por lesões traumáticas nessas estruturas. O trabalho foi desenvolvido com base nos protocolos e diretrizes do PRISMA, cuja metodologia envolveu um levantamento bibliográfico de estudos publicados nos últimos 10 anos, encontrados nas seguintes bases de dados da área da saúde: PubMed/Medline, Scopus, LILACS, SciELO e Cocrhane. Constatou-se que as implicações anatômicas das estruturas relacionadas ao corpo adiposo da bochecha são importantes para evitar o desenvolvimento de complicações após a retirada da bola de Bichat. Esses achados permitem concluir que é necessário entender o impacto que as complicações decorrentes de injúrias à estruturas anatômicas vizinhas ao corpo adiposo da bochecha podem ocasionar na qualidade de vida dos pacientes.

Palavras-chave: Estética; Procedimentos cirúrgicos bucais; Complicações intraoperatórias.

\begin{abstract}
This literature review aimed to study the complications resulting from the surgical removal of the buccal fat pad, seeking to understand the anatomical relationships between the structures involved in this procedure and to observe the main sequelae caused by traumatic injuries to these structures. The work was developed based on PRISMA protocols and guidelines, whose methodology involved a bibliographic survey of studies published in the last 10 years, found in the following databases in the health area: PubMed/Medline, Scopus, LILACS, SciELO and Cocrhane. It was found that the anatomical implications of structures related to the buccal fat pad are important to avoid the development of complications after the removal of the Bichat's fat pad. These findings allow us to conclude that it is necessary to understand the impact that complications resulting from injuries to anatomical structures adjacent to the buccal fat pad can have on the quality of life of patients.
\end{abstract}

Keywords: Esthetics; Oral surgical procedures; Intraoperative complications.

\section{Resumen}

Esta revisión de la literatura tuvo como objetivo estudiar las complicaciones que resultan de la extirpación quirúrgica del cuerpo adiposo de la mejilla, buscando comprender las relaciones anatómicas entre las estructuras involucradas en este procedimiento y observar las principales secuelas que provocan las lesiones traumáticas de estas estructuras. El trabajo se desarrolló en base a los protocolos y guías PRISMA, cuya metodología implicó un levantamiento bibliográfico de estudios publicados en los últimos 10 años, encontrados en las siguientes bases de datos del área de salud: PubMed / Medline, Scopus, LILACS, SciELO y Cocrhane. Se encontró que las implicaciones anatómicas de las estructuras relacionadas con el cuerpo adiposo de la mejilla son importantes para evitar el desarrollo de 
complicaciones luego de la remoción de la bola de Bichat. Estos hallazgos permiten concluir que es necesario comprender el impacto que las complicaciones derivadas de lesiones en estructuras anatómicas adyacentes al cuerpo adiposo de la mejilla pueden tener sobre la calidad de vida de los pacientes.

Palabras clave: Estética; Procedimientos quirúrgicos orales; Complicaciones intraoperatorias.

\section{Introdução}

A remoção d corpo adiposo da bochecha, ou bola de Bichat é um procedimento cirúrgico que vem se difundido mundialmente nos tempos atuais, sendo um assunto que merece destaque, já que entender as características dessa estrutura anatômica é um passo importante para a sua abordagem cirúrgica. Com base nisso, a bola de Bichat nada mais é do que uma massa de gordura biconvexa esférica circundada por uma camada fina de tecido conjuntivo, que por sua vez forma uma limitação capsular, sendo é encontrada bilateralmente na região maxilofacial. É uma extensão anterior do corpo adiposo mastigatório que preenche o espaço da musculatura mastigatória. Ele corresponde a um corpo principal e mais três extensões: o bucal, que é o principal, o pterigóide, temporal superficial e temporal profundo. Seu corpo principal é composto por três lobos: O lobo anterior se projeta na frente da borda anterior do músculo masseter; o intermediário se estende entre os músculos masseter e o bucinador; e o lobo posterior continua entre o espaço mastigatório temporal. Por essa razão, o corpo adiposo da bochecha está intimamente relacionado com o sistema mastigatório como um todo, com a glândula parótida, o nervo e artérias faciais. (Montero et al., 2018; Moura et al., 2018; Traboulsi-Garet et al., 2021).

Acreditava-se que essa estrutura anatômica não desempenhava função alguma na região facial. Entretanto, esse ponto e vista tem mudado, e acredita-se que a bola de Bichat afeta diretamente o volume interno da cavidade oral, além de auxiliar na função mastigatória, especialmente em bebês que se alimentam a partir da sucção. Além disso, ela atualmente é bastante utilizada em cirurgias reconstrutivas para defeitos intraorais. Esse tecido adiposo tende a diminuir com a idade, porém, em alguns casos, ele persiste em manter um volume que pode afetar a estética facial. Nesses casos, existe a indicação da excisão cirúrgica dessa estrutura para diminui o aspecto de inchaço do terço médio da face, destacar a proeminência do arco zigomático e do corpo mandibular, melhorando o aspecto facial. (Matarasso, 1991; Montero et al., 2018; Traboulsi-Garet et al., 2021).

Assim, para realizar corretamente a retirada do corpo adiposo da bochecha é necessário entender como ocorre a sua interação com as demais estruturas faciais. Os estudos demonstram uma grande variabilidade anatômica entre a distribuição dos ramos do nervo facial, o posicionamento do ducto da glândula parótida e a localização de ramos da artéria facial em relação à inserção do corpo adiposo da bochecha. Essas variações reiteram a importância em conhecer e entender as relações que existem entre a bola de Bichat e as demais estruturas próximas a ela, já que nos casos em que for feita sua excisão cirúrgica, essas estruturas devem ser separadas ilesas do corpo adiposo. Nesse sentido, a dissecação às cegas do corpo adiposo da bochecha pode acarretar em lesões no ramo bucal do nervo facial e da artéria facial, e também lesionar o ducto da glândula parótida, formando hematomas, abscessos, trismo e paralisia facial. (Benjamin \& Reish, 2018; Hwang et al., 2005).

Em relação ao exposto anteriormente, o objetivo deste trabalho é identificar as complicações que decorrem da retirada cirúrgica do corpo adiposo da bochecha, buscando entender as relações anatômicas entre as estruturas envolvidas nesse procedimento e observar as principais sequelas acarretadas por lesões traumáticas nessas estruturas.

\section{Metodologia}

A presente revisão de literatura foi feita tendo como base um checklist com as diretrizes do PRISMA, e que teve como objetivo nortear as etapas metodológicas do estudo, guiando o desenvolvimento da revisão. (Moher et al., 2009; Hermont et al., 2021). Pensando nisso, foi necessário estabelecer critérios de seleção para os artigos que seriam avaliados durante as etapas 
seguintes. Os critérios de inclusão escolhidos foram: artigos publicados entre janeiro de 2011 e junho de 2021; estudos escritos na língua portuguesa, inglesa e espanhola; trabalhos que tratem das implicações clínicas e cirúrgicas da remoção do corpo adiposo da bochecha; bem como aqueles que objetivam mostrar as complicações que podem ocorrer após a excisão da bola de Bichat. Além disso, alguns critérios foram selecionados para exclusão artigos que não sejam de interesse para esta revisão, são eles: estudos não disponíveis integralmente para leitura; revisões de literatura; trabalhos que não abordem as estruturas anatômicas envolvidas com o corpo adiposo da bochecha; artigos que tratem de procedimentos cirúrgicos específicos sem abordar a questão da anatomia da estrutura anatômica em questão.

Utilizando-se desses critérios, foi realizada uma busca eletrônica dentro das bases de dados da área da saúde PubMed/Medline, Scopus, LILACS, SciELO e Cochrane. Para realizar essa busca foi necessário desenvolver uma estratégia de busca com base em descritores Decs/ MeSH, e termos alternativos unidos a operadores booleanos. A seguinte estratégia doi utilizada para esta revisão: "Buccal Fat Pad" AND "Surgery, Plastic". A disposição desses termos retornou os resultados dos estudos que incluíram termos semelhantes em seus trabalhos. Com os artigos dispostos, o levantamento bibliográfico inicial foi feito com base na leitura dos títulos e resumos dos artigos, selecionando os que atenderam aos critérios estabelecidos anteriormente. Após isso, uma pesquisa manual das referências dos estudos presentes nas bases de dados foi feita, para complementar a seleção dos artigos. No fim, foram excluídos os estudos duplicados e os que não se encaixavam no perfil de critérios. Os artigos finais foram analisados a partir de uma tabela de resumo, para auxiliar a transcrição dos dados de cada estudo.

\section{Resultados}

A estratégia de busca eletrônica dos trabalhos resultou em um total de 127 estudos em todas as bases de dados pesquisadas. Após a leitura dos títulos e dos resumos, restaram 05 artigos de interesse para o objetivo deste trabalho. Deste número, 01 artigo foi excluído, pois não pôde ser obtido na íntegra para leitura, restando um total de 04 artigos para a avaliação completa de seu conteúdo. Outros 04 trabalhos foram adicionados com base em uma busca manual de referências dos que já estavam previamente selecionados. Com base nisso, foi realizado com base em 08 artigos selecionados entre os critérios estabelecidos. A seleção dos estudos está ilustrada no fluxograma adaptado com base nas diretrizes do PRISMA (Figura 1). 
Figura 1 - Fluxograma adaptado do PRISMA statement for systematic reviews.

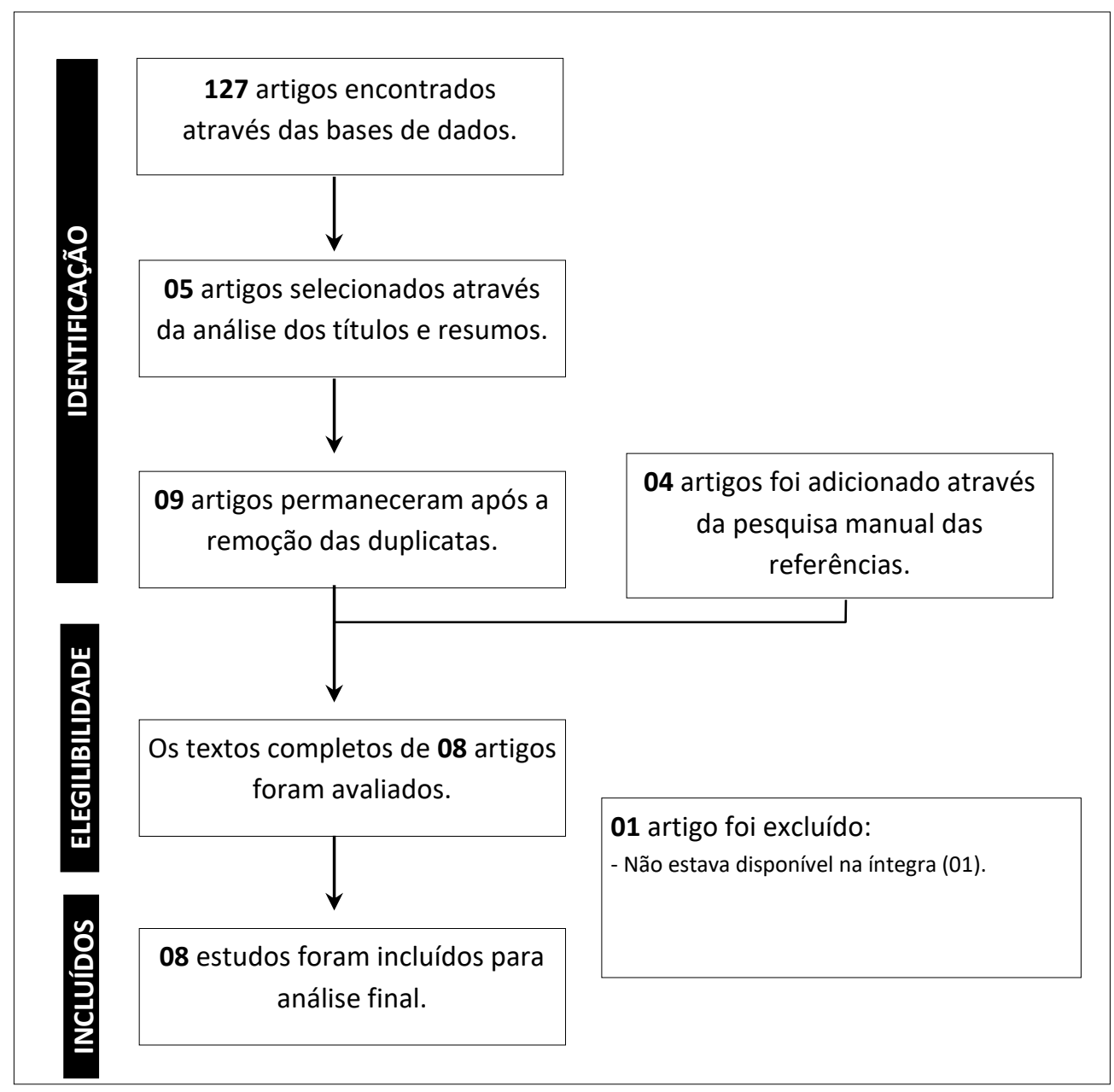

Fonte: Moher et al. (2009).

Com o objetivo de auxiliar a análise dos textos, um resumo metodológico dos principais achados e conclusões dos estudos selecionados está presente abaixo na Tabela 1. O estudo de Engdahl et al., (2012) acompanhou e tratou uma complicação de alto risco após a retirada do corpo adiposo da bochecha. O paciente deu entrada com um sangramento grave e persistente em profundidade dentro do espaço bucal, com riscos de ocorrência de choque hemorrágico. Traumas vasculares massivos geralmente não são reportados em casos de retiradas do corpo adiposo da bochecha. Entretanto, essa estrutura anatômica está interligada a ramos da artéria maxilar interna, artérias temporais superficiais e as artérias faciais. Nesse caso específico, ocorreu a dissecação profunda da bola de Bichat, lesionando ramos da artéria maxilar interna, causando o grave sangramento com risco de morte.

Recentemente Hernández et al., (2021) avaliaram os conhecimentos sobre o corpo adiposo da bochecha, ou bola de Bichat, e as estruturas associadas a ele, levando em consideração um caso de complicação cirúrgica após excisão cirúrgica da estrutura anatômica. O caso clínico em questão apresentou um paciente que, após a remoção cirúrgica da bola de Bichat, desenvolveu um abscesso bucal esquerdo quatro dias após o procedimento cirúrgico. Essa complicação ocorreu devido a uma obstrução traumática do ducto parotídeo, seccionando-o durante o procedimento. O ducto parotídeo se relaciona de três diferentes formas com o corpo adiposo da bochecha, podendo se encontrar superficialmente, acima ou dentro do corpo, sendo este último associado ao caso reportado de secção. 
Anteriormente, Kuppel et al., (2018) analisaram a anatomia do corpo adiposo da bochecha, as aplicações clínicas dessa estrutura e as possíveis complicações cirúrgicas e pós-cirúrgicas após a remoção dessa estrutura anatômica, tudo isso através de relatos de uma série de casos de remoção cirúrgica da bola de Bichat. Os casos relatados de complicações foram variados, incluindo três diferentes situações de injúria após a remoção cirúrgica do corpo adiposo da bochecha. O primeiro caso foi o desenvolvimento de um abscesso causado por uma lesão do ducto parotídeo que acarretou em retenção do fluido salivar. O segundo caso de complicação se manifestou através de necrose tecidual e hematoma pós-operatório gerados por um acesso cirúrgico inadequado e possível lesão nos ramos da artéria facial. Por último, um outro caso de complicação foi relatado em que o paciente apresentou alterações na função muscular perioral, produzido por uma lesão do ramo bucal do nervo facial, comprometendo a função estética e originando parestesia nos músculos acometidos.

Um outro estudo buscou enfatizar a relação anatômica entre o corpo adiposo da bochecha e os ramos terminais do nervo facial. Assim, Porto et al., (2020) descobriram que esses ramos nervosos possuem padrões diferentes entre os indivíduos, porém, esses ramos terminais sempre estão próximos e lateralmente posicionados ao corpo adiposo a bochecha. Dessa forma, foram encontrados três padrões e ramos diferentes passando próximos à estrutura anatômica em questão. Essa complexidade anatômica do sistema nervoso pode causar alto risco de os pacientes desenvolverem paralisia temporária ou permanente desses ramos nervosos como sequelas pós-cirúrgicas.

Logo após, Rohrich et al., (2021) avaliaram se a excisão cirúrgica do corpo adiposo da bochecha poderia causar envelhecimento precoce e distorção do terço médio da face ao longo do tempo. Os resultados demonstraram que se a excisão do corpo adiposo da bochecha se limitar apenas a sua extensão bucal, ocorrerá risco mínimo para o envelhecimento prematuro da face. Por essa razão, é fundamental que o cirurgião seja cuidado, evitando a ressecção excessiva, que acarretaria em uma distorção do terço médio facial e consequente envelhecimento facial precoce.

Enquanto isso, Roman-Torres et al., (2021) observaram o pós-operatório de excisões do corpo adiposo da bochecha, para determinar possíveis complicações e defeitos. Todos os casos relataram sentir limitação de abertura bucal, dor local e edema facial bilateral nos primeiros 10 dias após o procedimento cirúrgico. Três casos relataram aumento do edema facial, ocasionados pela falta de descanso e não cumprimento das recomendações médicas. Já a limitação da abertura de boca regressou aos passar dos dias, voltando a abertura inicial antes da cirurgia por volta do trigésimo dia de pós-operatório.

Por outro lado, Surek et al., (2021) examinaram detalhadamente a anatomia do corpo adiposo da bochecha para encontrar abordagens cirúrgicas seguras que evitem complicações posteriores. Entendendo que anatomicamente o corpo adiposo da bochecha tem alta proximidade com o ducto parotídeo e os ramos bucais do nervo facial, identificar precisamente a localização do corpo adiposo antes da incisão da cápsula ajuda a evitar complicações indesejadas. Quando a cápsula que reveste a bola de Bichat é incisada, as estruturas relacionadas essas estruturas retraem para a cápsula, fornecendo uma zona segura para a excisão cirúrgica. Dessa forma, se a anatomia local for entendida e as estruturas pertinentes forem evitadas, levando em consideração a existência de variações anatômicas específicas de cada paciente, é possível realizar uma técnica cirúrgica de forma simples e segura, evitando a ocorrência de hematomas, infecções, trismo e injúrias em nervos e ductos.

Por fim, Vieira et al., (2019) descreveram um caso em que a cirurgia de retirada do corpo adiposo da bochecha resultou em danos na glândula parótida e artéria bucal. O paciente apresentou um quadro clínico de edema bilateral no terço médio da face, se estendendo para a parte inferior, dor à palpação, trismo moderado, e equimoses infraorbital, bucal e cervicais. Logo após, o paciente foi diagnosticado com uma lesão na glândula parótida, com a presença de coleção serosa. Além disso, o paciente também apresentou uma lesão na artéria bucal direita, que evoluiu para um processo infeccioso devido à presença de hematomas. 
Tabela 1 - Sumarização dos resultados.

\begin{tabular}{|c|c|c|}
\hline Autor/ Ano & Amostra & Principais dados encontrados \\
\hline $\begin{array}{l}\text { Engdahl et } \\
\text { al., } 2012\end{array}$ & 01 paciente. & $\begin{array}{l}\text { O caso mostrou uma dissecação profunda } \\
\text { da bola de Bichat, lesionando ramos da } \\
\text { artéria maxilar interna, causando o grave } \\
\text { sangramento com risco de morte. }\end{array}$ \\
\hline $\begin{array}{l}\text { Hernández } \\
\text { et al., } 2021\end{array}$ & 01 paciente. & $\begin{array}{l}\text { Paciente apresentou um abscesso como } \\
\text { complicação após cirurgia de remoção do } \\
\text { corpo adiposo da bochecha ocasionada por } \\
\text { secção acidental do ducto parotídeo, que } \\
\text { estava localizado internamente ao corpo } \\
\text { adiposo. }\end{array}$ \\
\hline $\begin{array}{l}\text { Kluppel et } \\
\text { al., } 2018\end{array}$ & 03 pacientes. & $\begin{array}{l}\text { Três casos de complicações foram } \\
\text { avaliados: o primeiro foi a presença de um } \\
\text { abscesso causado por lesão do ducto } \\
\text { parotídeo; o segundo foi necrose tecidual e } \\
\text { hematoma pós-cirúrgico ocasionados por } \\
\text { acesso cirúrgico inadequado e possível } \\
\text { lesão na artéria facial; a última } \\
\text { complicação foi o desenvolvimento de } \\
\text { parestesia nos músculos periorais por lesão } \\
\text { do nervo facial. }\end{array}$ \\
\hline $\begin{array}{l}\text { Porto et al., } \\
2020\end{array}$ & $\begin{array}{l}03 \text { hemifaces de } \\
\text { cadáveres humanos. }\end{array}$ & $\begin{array}{l}\text { As amostras exibiram variações nos } \\
\text { padrões de distribuição dos ramos } \\
\text { terminais nervosos do nervo facial, porém, } \\
\text { todas as terminações passam próximos e } \\
\text { lateralmente ao corpo adiposo da } \\
\text { bochecha. }\end{array}$ \\
\hline
\end{tabular}

\begin{tabular}{|c|c|c|c|}
\hline $\begin{array}{l}\text { Rohrich et } \\
\text { al., } 2021\end{array}$ & $\begin{array}{l}2 \text { corpos adiposos da } \\
\text { bochecha de um cadáver } \\
\text { humano. }\end{array}$ & $\begin{array}{l}\text { A excisão cirúrgica controlada da extensão } \\
\text { bucal do corpo adiposo da bochecha } \\
\text { diminui as chances de ocorrer distorções } \\
\text { do terço médio da face e, } \\
\text { consequentemente, envelhecimento facial } \\
\text { precoce. }\end{array}$ & $\begin{array}{l}\text { A retirada cirúrgica da bola de Bichat } \\
\text { para fins estéticos ainda é uma área de } \\
\text { muito debate, já que podem causar } \\
\text { riscos com o passar do tempo. No } \\
\text { entanto, foi possível observar que } \\
\text { quando a remoção é feita com tração } \\
\text { suave da extensão bucal do corpo } \\
\text { adiposo, os riscos de causar distorção } \\
\text { facial são diminuídos. }\end{array}$ \\
\hline $\begin{array}{l}\text { Roman- } \\
\text { Torres et al., } \\
2020\end{array}$ & 40 pacientes. & $\begin{array}{l}\text { Todos os casos relataram sentir limitação } \\
\text { de abertura bucal, dor local e edema facial } \\
\text { bilateral nos primeiros } 10 \text { dias após o } \\
\text { procedimento cirúrgico. Três casos } \\
\text { relataram aumento do edema facial, } \\
\text { ocasionados pela falta de descanso e não } \\
\text { cumprimento das recomendações médicas. }\end{array}$ & $\begin{array}{l}\text { A presença de trismo, hemorragias } \\
\text { e assimetria facial ocorreram por } \\
\text { atividade física excessiva após o } \\
\text { procedimento, bem como a falta de } \\
\text { comprometimento com as a a } \\
\text { recomendaçes médicas. }\end{array}$ \\
\hline $\begin{array}{l}\text { Surek et al., } \\
2021\end{array}$ & $\begin{array}{l}18 \text { hemifaces de } \\
\text { cadáveres humanos. }\end{array}$ & $\begin{array}{l}\text { O corpo adiposo da bochecha tem alta } \\
\text { proximidade com o ducto parotídeo e os } \\
\text { ramos bucais do nervo facial, identificar } \\
\text { precisamente a localização do corpo } \\
\text { adiposo antes da incisão da cápsula ajuda a } \\
\text { evitar complicações indesejadas. }\end{array}$ & $\begin{array}{l}\text { Entender a anatomia, as medidas e os } \\
\text { pontos de referência das estruturas } \\
\text { anatômicas associadas ao corpo } \\
\text { adiposo da bochecha ajudam os } \\
\text { cirurgiões a identificar a extensão do } \\
\text { corpo adiposo. }\end{array}$ \\
\hline $\begin{array}{l}\text { Vieira et al., } \\
2019\end{array}$ & 01 paciente. & $\begin{array}{l}\text { O paciente apresentou um quadro clínico } \\
\text { de edema bilateral no terço médio da face, } \\
\text { se estendendo para a parte inferior, dor à } \\
\text { palpação, trismo moderado, e equimoses } \\
\text { infraorbital, bucal e cervicais. O } \\
\text { diagnóstico foi de lesão da glândula } \\
\text { parótida e artéria bucal. }\end{array}$ & $\begin{array}{l}\text { A excisão cirúrgica do corpo adiposo } \\
\text { da bochecha é um procedimento } \\
\text { estético, mas pode causar várias } \\
\text { lesões em estruturas nobres próximas, } \\
\text { podendo acarretar em lesões com } \\
\text { efeitos colaterais irreversíveis. }\end{array}$ \\
\hline
\end{tabular}

Fonte: Autores (2021).

\section{Discussão}

Os estudos deixam claro que procedimentos cirúrgicos realizados sem o devido conhecimento anatômico são suscetíveis a erros e assim, ao surgimento de complicações pós-operatórias graves para a saúde do paciente. A retirada cirúrgica do corpo adiposo da bochecha, apesar de ser considerado um procedimento simples, pode acarretar no desenvolvimento de complicações, pois essa estrutura está em contato íntimo com outras importantes estruturas faciais. De 
modo geral, Roman-Torres et al., (2020) relataram pequenas intercorrências pós-cirúrgicas em excisões do corpo adiposo da bochecha. Assim, normalmente os pacientes relatam limitações na abertura bucal, dor localizada e edema facial durante os 10 primeiros dias após as cirurgias, com resolução completa em até 30 dias. Além disso, essas intercorrências em muitos casos estão associadas a falta de descanso após o procedimento e ausência do cumprimento das recomendações médicas, sendo assim, cirurgias de baixa lesividade, quando não afetam as estruturas vizinhas.

O estudo de Surek et al., (2021) comprovou a alta proximidade anatômica do corpo adiposo da bochecha com o ducto parotídeo e os ramos bucais do nervo facial, ratificando a relevância em identificar precisamente a localização do corpo adiposo antes da incisão da cápsula que a reveste, ajudando a evitar complicações indesejadas. Entretanto, caso não seja se tome os cuidados necessários com as variações anatômicas entre as estruturas faciais, aumentam-se os riscos do surgimento de hematomas, infecções, trismo e injúrias nos nervos e ductos.

A glândula parótida se relaciona de três maneiras com o corpo adiposo da bochecha, e é extremamente importante entender essa relação para que não a danifique durante o procedimento cirúrgico. O ducto parotídeo pode estar em contato pode estar em contato com a bola de Bichat superficialmente, em posição superior ou até mesmo imerso dentro do corpo adiposo, sendo esta última disposição anatômica a de maior risco de dano. (Hwang et al., 2005). Com base nisso, Hernández et al., (2021) observaram um caso de desenvolvimento de um abscesso facial após um procedimento cirúrgico de remoção da bola de Bichat, sendo ocasionada devido a uma obstrução traumática do ducto parotídeo, que foi seccionado durante a cirurgia. Essa mesma complicação associada ao seccionamento do ducto parotídeo foi observada nos estudos de Kluppel et al., (2018) e Vieira et al., (2019), deixando claro o desenvolvimento de lesões que necessitarão ser tratadas, mas com possibilidade de deixar sequelas permanentes.

Como visto anteriormente, além da comunicação anatômica com o ducto parotídeo, o corpo adiposo da bochecha ainda se relaciona com ramos bucais do nervo facial. Pensando nisso, Porto et al., (2020) observaram que esses ramos nervosos possuem diferentes padrões que variam entre indivíduos. Entretanto, apesar dessa abrangente variação anatômica, os ramos da do nervo facial, porém, se encontram em posição lateral ao corpo adiposo a bochecha, com alta proximidade entre os dois. Por essa razão, essa outra estrutura também possui alto risco de ser lesionada durante a remoção da bola de Bichat. O estudo de Kluppel et al., (2018) expôs um caso de complicação em que o paciente desenvolveu parestesia dos músculos periorais por uma lesão do ramo bucal do nervo facial, comprometendo a função estética dessa paciente, sendo que essa sequela pode se tornar irreversível.

As duas estruturas abordadas representam maioria dos casos relatados na literatura de complicações pós-operatórias da retirada do corpo adiposo da bochecha. Todavia, a presença de ramos arteriais próximos a essa estrutura também torna possível a ocorrência de lesões nesses ramos durante o processo de retirada da bola de Bichat. Kluppel et al., (2018) relataram que as lesões nos ramos da artéria facial ocasionaram a presença de hematomas pós-operatórios, que tiveram resolução total com o passar do tempo. Esse tipo de lesão arterial, em geral, não acarretam em problemas graves. Entretanto, Engdahl et al., (2012) acompanharam um caso de sangramento grave e persistente em profundidade dentro do espaço bucal, com risco de choque hemorrágico. Um trauma vascular massivo como esse geralmente não ocorre em um procedimento de excisão do corpo adiposo da bochecha, mas nesse caso específico, foi feita uma dissecação profunda da bola de Bichat, que casou lesões nos ramos da artéria maxilar interna, trazendo riscos sérios à vida do paciente.

Além dessas lesões às estruturas faciais vizinhas ao corpo adiposo da bochecha, a sua excisão pode causar ainda complicações estéticas. Essas alterações são confirmadas por Rohrich et al., (2021), demonstrando que excisões que ultrapassem a extensão bucal do corpo adiposo da bochecha podem aumentar o risco de envelhecimento prematuro da face e distorção do terço médio facial. Por essa razão, cirurgias estéticas de remoção da bola de Bichat devem se restringir a essa extensão bucal, evitando a sua ressecção excessiva, e os problemas estéticos futuros que essas abordagens podem ocasionar. 
Com base nas informações literárias, a retirada do corpo adiposo da bochecha, apesar de ser um procedimento considerado básico e simples, e que está altamente difundido na realidade dos atendimentos odontológicos, exige do profissional da odontologia um bom conhecimento da sua anatomia. Por essa razão, existe uma necessidade de estar atento às relações anatômicas entre o corpo adiposo da bochecha e as estruturas faciais relacionadas a ele, com o intuito de se evitar injúrias durante as cirurgias. (Hernández et al., 2021; Surek et al., 2021).

\section{Conclusão}

O procedimento de retirada do corpo adiposo da bochecha, na maior parte dos casos, acontece com uma finalidade estética. Essa estrutura anatômica se relaciona diretamente ao ducto da glândula parótida, aos ramos terminais do nervo facial e da artéria facial. A proximidade com essas estruturas pode causar complicações pós-cirúrgicas, nos casos em que o cirurgião não possui um bom conhecimento anatômico. As complicações envolvidas em cirurgias de remoção da bola de Bichat envolvem o desenvolvimento de abscessos, hematomas, sangramentos, paralisias faciais e distorções estéticas do terço médio da face. Essas complicações podem causar sequelas irreversíveis. Com isso, o cuidado com as estruturas associadas ao corpo adiposo da bochecha evita complicações cirúrgicas que afetam a qualidade de vida dos pacientes.

\section{Referências}

Benjamin, M., \& Reish, R. G. (2018). Buccal Fat Pad Excision: Proceed with Caution. PRS Global Open, 1 (1), 1-4.

Engdahl, R., Nassiri, N., Mina, B., Drury, J., \& Rosen, R. (2012). Superselective Microcatheter Embolization of Hemorrhage after Buccal Lipectomy. Aesth Plast Surg, 36 (1), 742-745.

Hermont, A. P., Zina, L. G., Silva, K. D., Silva, J. M., \& Martins-Júnior, P. A. (2021). Revisões integrativas: conceitos, planejamento e execução. Arq Odontol, 57 (1), 3-7.

Hernández, O., Altamirano, J., Soto, R., \& Rivera, A. (2021). Relaciones Anatómicas del Cuerpo Adiposo de la Mejilla Asociadas a Complicaciones de bichectomía. A Propósito de un Caso. Int. J. Morphol., 39 (1), 123-133.

Hwang, K., Cho, H. J., Battuvshin, D., Chung, I. H., \& Hwang, S. H. (2005). Interrelated Buccal Fat Pad With Facial Buccal Branches and Parotid Duct. The Journal Of Craniofacial Surgery, 16 (4), 658-660.

Kluppel, L., Marcos, R. B., Shimizu, I. A., Silva, M. A. D., \& Silva, R. D. (2018). Complications associated with the bichectomy surgery. Rev Gaúch Odontol., 66 (3), 278-284.

Matarasso, A. (1991). Buccal Fat Pad Excision: Aesthetic Improvement os the Midface. Ann Plast Surg, 26 (1), $413-418$.

Moher, D., Liberati, A., Tetzlaff, J., Altman, D. G., \& The PRISMA Group. (2009). Preferred Reporting Items for Systematic Reviews and Meta-Analyses: The PRISMA Statement. Plos Medicine, 6 (7), 1-6.

Montero, J. F. D., Souza, H. C. M., Martins, M. S., Oliveira, M. N., Benfatti, C. A. M., \& Magini, R. S. (2018). Versatility and Importance of Bichat's Fat Pad in Dentistry: Case Reports of Its Use in Occlusal Trauma. J Contemp Dent Pract, 19 (7), 888-894.

Moura, L. B., Spin, J. R., Spin-Neto, R., \& Pereira-Filho, V. A. (2018). Buccal fat pad removal to improve facial aesthetics: an established technique? Med Oral Patol Oral Cir Bucal, 23 (4), 1-7.

Porto, L. B., Nazer, M. B., \& Piazza, J. L. (2020). Relação Anatômica da Bola de Bichat com Ramos Terminais do Nervo Facial. Rev. Cir. Traumatol. BucoMaxilo-Fac., 20 (4), 12-15.

Rohrich, R. J., Stuzin, J. M., Satesky, I. L., Avashia, Y. J., Agrawal, N. A., \& Prada, M. (2021). The Role of the Buccal Fat Pad in Facial Aesthetic Surgery. Plastic and Reconstructive Surgery, 148 (2), 334-338.

Roman-Torres, C., Domingues, N. R. A. P., Pimentel, A. C., Marão, H. F., \& Sendyk, W. R. (2020). Post-Operative Evaluation of the Intra-Oral Buccal Fat Pad Removal Technique: A Prospective Study. The Open Dentistry Journal, 14 (1), 324-328.

Surek, C. C., Kochuba, A. L., Said, S. A., Cho, K. H., Swanson, M., Duraes, E., McBride, J., Drake, R. L., \& Zins, J. E. (2021). External Approach to Buccal Fat Excision in Facelift: Anatomy and Technique. Aesthetic Surgery Journal, 4 (5), 527-534.

Traboulsi-Garet, B., Camps-Font, O., Traboulsi-Garet, M., \& Gay-Escoda, C. (2021). Buccal fat pad excision for cheek refinement: A systematic review. Med Oral Patol Oral Cir Bucal., 26 (4), 474-481.

Vieira, G. M., Jorge, F. D., Franco, E. J., Dias, L. C., Guimarães, M. C. M., \& Oliveira, L. A. (2019). Lesions of the Parotid Gland and Buccal Artery After Buccal Fat Pad Reduction. The Journal of Craniofacial Surgery, 30 (3), 790-792. 